\title{
GXI-conjugated poly(lactic acid) nanoparticles encapsulating Endostar for improved in vivo anticolorectal cancer treatment
}

This article was published in the following Dove Press journal:

International Journal of Nanomedicine

28 May 2015

Number of times this article has been viewed

\author{
Yang $\mathrm{Du}^{1, *}$ \\ Qian Zhangl,* \\ Lijia Jing ${ }^{2}$ \\ Xiaolong Liang ${ }^{2}$ \\ Chongwei Chi' \\ Yaqian $\mathrm{Li}^{\prime}$ \\ Xin Yang' \\ Zhifei Dai ${ }^{2}$ \\ Jie Tian'
}

'Key Laboratory of Molecular Imaging, The State Key Laboratory of Management and Control for

Complex Systems, Institute of Automation, Chinese Academy of Sciences, Beijing, People's Republic of China; ${ }^{2}$ Department of Biomedical Engineering, College of Engineering, Peking University, Beijing People's Republic of China

*These authors contributed equally to this work
Correspondence: Jie Tian

Key Laboratory of Molecular Imaging,

The State Key Laboratory of

Management and Control for Complex

Systems, Institute of Automation,

Chinese Academy of Sciences, 95

ZhongGuanCun East Road, 100190

Beijing, People's Republic of China

Tel +86 I0 62527995

Fax +86 I0 62527995

Email jie.tian@ia.ac.cn

\begin{abstract}
Tumor angiogenesis plays a key role in tumor growth and metastasis; thus, targeting tumor-associated angiogenesis is an important goal in cancer therapy. However, the efficient delivery of drugs to tumors remains a key issue in antiangiogenesis therapy. GX1, a peptide identified by phage-display technology, is a novel tumor vasculature endothelium-specific ligand and possesses great potential as a targeted vector and antiangiogenic agent in the diagnosis and treatment of human cancers. Endostar, a novel recombinant human endostatin, has been shown to inhibit tumor angiogenesis. In this study, we developed a theranostic agent composed of GX1-conjugated poly(lactic acid) nanoparticles encapsulating Endostar (GPENs) and labeled with the near-infrared dye IRDye $800 \mathrm{CW}$ to improve colorectal tumor targeting and treatment efficacy in vivo. The in vivo fluorescence molecular imaging data showed that GPENs (IRDye 800CW) more specifically targeted tumors than free IRDye 800CW in colorectal tumor-bearing mice. Moreover, the antitumor efficacy was evaluated by bioluminescence imaging and immunohistology, revealing that GPENs possessed improved antitumor efficacy on subcutaneous colorectal xenografts compared to other treatment groups. Thus, our study showed that GPENs, a novel GX1 peptide guided form of nanoscale Endostar, can be used as a theranostic agent to facilitate more efficient targeted therapy and enable real-time monitoring of therapeutic efficacy in vivo.
\end{abstract}

Keywords: GX1 peptide, Endostar, colorectal cancer, angiogenesis, IRDye 800CW, molecular imaging

\section{Introduction}

Colorectal cancer (CRC) is the second most common malignancy in the western world. Despite recent advances in chemotherapeutics, this disease continues to be one of the leading causes of cancer-related morbidity and mortality in the United States. Targeted detection and therapy using recently identified in vivo cancer biomarkers are likely to result in significant improvements in methods for early detection, risk stratification, and therapeutic intervention. ${ }^{1-3}$

Tumor angiogenesis plays a key role in tumor growth and metastasis; thus, targeting angiogenesis is one method to inhibit cancer progression. ${ }^{4,5}$ Tumor vasculature undergoing angiogenesis expresses specific endothelial surface markers (vascular zip codes), which are absent or seldom detectable in mature vessels. Targeting tumor-associated angiogenesis is an important goal in cancer therapy; however, the efficient delivery of drugs to the endothelial cells of the tumor vasculature remains an important issue in antiangiogenesis therapy. ${ }^{6,7}$ Numerous targeting moieties have been used for target-specific delivery of antiangiogenesis therapies, including small 
molecules, peptides, proteins, and antibodies. ${ }^{8-10}$ Among these targeting moieties, small peptides with a low molecular weight demonstrate a number of distinct advantages over other targeting strategies. In general, small peptides are highly permeable, permitting rapid access to target tissues. ${ }^{11}$ Furthermore, peptides usually have low toxicity and immunogenicity and can be chemically modified flexibly. ${ }^{12,13}$ The GX1 peptide (CGNSNPKSC), identified by phage-display technology, has been demonstrated as a tumor vasculature endothelium-specific ligand. ${ }^{14-16}$ Previous studies pointed out that the GX1 peptide plays a role in gastric cancer-associated angiogenesis, suggesting that GX1 peptide-based assays may be one method to image tumor angiogenesis to improve diagnosis and therapy.

Endostar, a novel recombinant human endostatin expressed in and purified from Escherichia coli, was approved by the Chinese State Food and Drug Administration in 2005 for the treatment of non-small-cell lung cancer. Recent studies have demonstrated that Endostar combined with chemotherapy can improve treatment efficacy and inhibit disease progression in advanced CRC patients. ${ }^{17,18}$ However, despite the apparent therapeutic value of Endostar, its biological halflife, similar to most protein-based drugs, is short owing to rapid metabolism. ${ }^{19}$ Therefore, a long-acting formulation of Endostar could provide stable antitumor activity and fewer injection-related complications.

The ability to create agents incorporating multiple functionalities, such as targeting, imaging, and therapy, is one of the major advantages of nanoparticles. Particularly, the combination of therapeutic and diagnostic moieties into theranostic nanosystems confers unique capabilities. ${ }^{20,21}$ Previous work has shown the specific tumor endothelial targeting effects of GX1 in vivo using different imaging modalities. However, to the best of our knowledge, the combination of GX1 with antiangiogenesis therapeutic drugs has not been tested before.

Noninvasive molecular imaging enables cellular and physiological processes to be visualized and quantified in vivo and is therefore widely recognized as a tool for cancer detection in most organs. Molecular imaging can offer potential advantages over traditional biopsy-based procedures to predict and assess treatment response. ${ }^{22-24}$ Compared with other imaging modalities, such as nuclear imaging, optical molecular imaging is relatively inexpensive, sensitive, and straightforward, presenting an ideal imaging modality for improving our understanding of CRC. ${ }^{25,26}$ Optical molecular imaging may eventually lead to earlier diagnosis and better prognosis than conventional detection strategies. Biological tissues have lower absorbance and autofluorescence in the near-infrared (NIR) region (650-900 nm), which allows a relatively high signal-to-background ratio. ${ }^{27,28}$ The development of NIR fluorophores and nanomaterials has facilitated the noninvasive imaging of tumors at the molecular and cellular level in living subjects. ${ }^{29,30}$

The objective of this study was to develop theranostic nanoparticles, termed GX1-conjugated poly(lactic acid) nanoparticles encapsulating Endostar (GPENs), which would allow for specific tumor targeting, in vivo imaging, and improved drug therapeutic efficacy. Here, the GX1 peptide was conjugated to GPENs as an active target to the colorectal tumor vasculature, and the NIR dye IRDye $800 \mathrm{CW}$ was conjugated to the GPENs surface to monitor the biodistribution and tumor-targeting efficacy of nanoparticles through fluorescence molecular imaging (FMI). The GPENs were administered to subcutaneous colorectal tumor-bearing mice, and the tumor growth and treatment efficacy were dynamically examined by measuring the tumor bioluminescence imaging (BLI). At the end of the treatment period, the tumors were dissected for further histological evaluation to confirm the in vivo observations. Our study may provide an experimental basis for the clinical application of GPENs in the future.

\section{Materials and methods Materials}

D-Luciferin was purchased from Biotium (Fremont, CA, USA). Endostar was obtained from Shandong Simcere Medgeen Bio-Pharmaceutical Co Ltd (Nanjing, People's Republic of China). Polylactic acid (PLA) was purchased from the Medical Instrumental Institute (Shandong, People's Republic of China). IRDye $800 \mathrm{CW}$ was obtained from Li-COR Biosciences (Lincoln, NE, USA). The GX1 peptide was purchased from APeptide Co. Ltd. (Shanghai, People's Republic of China). The colon cancer cell line COLO205fLuc was provided by the Department of Radiology, Peking Union Medical College Hospital.

\section{Synthesis of GPENs}

PLA nanoparticles encapsulating Endostar (PENs) were prepared using an adapted water-in-oil-in-water $(\mathrm{W} / \mathrm{O} / \mathrm{W})$ double-emulsion solvent evaporation method. Briefly, PLA (60 mg) was dissolved in methylene chloride $(10 \mathrm{~mL})$. The first emulsion (W/O) was generated between the PLA solution and the Endostar solution ( $3 \mathrm{~mL}, 5 \mathrm{mg} / \mathrm{mL})$ by shearing (2,800 rpm for 60 seconds). Then, the first $\mathrm{W} / \mathrm{O}$ emulsion was poured into a PLA solution $(20 \mathrm{~mL}, 0.1 \% \mathrm{w} / \mathrm{v})$ and 
homogenized to obtain the double emulsion $(\mathrm{W} / \mathrm{O} / \mathrm{W})$ by high-speed shearing (25,000 rpm for 60 seconds). The W/O/W double emulsion was evaporated in an aqueous solution $(100 \mathrm{~mL})$ containing $0.1 \%$ polyvinyl alcohol $(\mathrm{w} / \mathrm{v})$ by gentle magnetic stirring at room temperature to ensure the methylene chloride was evaporated. The nanoparticles were collected by centrifugation $(15,300 \mathrm{rpm}$ for 60 minutes) and washed three times with phosphate-buffered saline (PBS), followed by lyophilization $\left(-54^{\circ} \mathrm{C}, 36\right.$ hours $)$ using a freeze dryer.

The dry PENs were redispersed in PBS buffer. IRDye $800 \mathrm{CW}(3 \mathrm{mg}$ ) and $120 \mu \mathrm{L}$ of enhanced carbodiimide/ $\mathrm{N}$-hydroxysuccinimide (equal concentrations at $10 \mathrm{mg} / \mathrm{mL}$ in PBS buffer) were added to the PENs solution (containing $300 \mathrm{mg}$ PENs) and gently stirred for 24 hours. The product was then isolated by centrifugation at $15,300 \mathrm{rpm}$ for $40 \mathrm{~min}$ utes, washed with PBS three times, and lyophilized.

The dry PENs-IRDye $800 \mathrm{CW}$ was redispersed in PBS buffer. GX1 peptide (4 mg) and $120 \mu \mathrm{L}$ of enhanced carbodiimide/ $N$-hydroxysuccinimide (equal concentrations at $10 \mathrm{mg} / \mathrm{mL}$ in PBS buffer) were added into the PENs-IRDye $800 \mathrm{CW}$ solution containing $300 \mathrm{mg}$ of nanoparticles and gently stirred for 24 hours. The product was then purified by centrifugation at $15,300 \mathrm{rpm}$ for 40 minutes, washed with PBS buffer three times, and lyophilized to obtain the final GPENs (IRDye 800CW) (Figure 1).

\section{Characterization of GPENs}

To characterize the nanoparticles, the typical morphology and size of the empty PLA-nanoparticles (PNPs) and GPENs were analyzed by scanning electron microscope (SEM, NANOSEM430, FEI, Hillsboro, OR, USA). Nanoparticle size distribution and zeta potentials were evaluated by a
90Plus/BI-MAS instrument (Brookhaven Instruments Co., Holtsville, NY, USA).

\section{In vitro drug release study}

The in vitro release of Endostar from GPENs was analyzed in PBS (pH 7.4 and pH 5.0). Briefly, $20 \mathrm{mg}$ of GPENs was dispersed in $5 \mathrm{~mL}$ of PBS. The sample solution was added to two dialysis bags with a molecular weight cut-off of $14,000 \mathrm{Da}$ and placed into a beaker containing $100 \mathrm{~mL}$ of PBS solution $(10 \mathrm{mM})$ with a varying $\mathrm{pH}$. The beaker was maintained at $37^{\circ} \mathrm{C}$ with gentle stirring. The amount of drug released into the media was determined by withdrawing samples at predetermined time intervals and then adding an equal volume of PBS to maintain the medium total volume. The protein concentration was measured using a Pierce Bicinchoninic acid Protein (BCA) assay (Thermo Scientific, Waltham, MA, USA). The release curve of Endostar from GPENs was then described.

In addition, to detecting the stability of the carriers, the change in GPEN diameter in mouse serum was examined over 24 hours. The GPENs $(0.068 \mathrm{nM})$ were suspended in $50 \%$ mouse serum $50 \%$ PBS and incubated at $37^{\circ} \mathrm{C}$ for 24 hours. Samples were collected 0 hour, 4 hours, 8 hours, and 24 hours after incubation. After centrifugation, the NPs were dispersed in PBS, and size distribution was evaluated by dynamic light scattering using a 90Plus/BI-MAS instrument.

\section{Cell culture}

COLO205-fLuc cells were cultured in Roswell Park Memorial Institute 1640 (HyClone, Thermo Scientific) and supplemented with $10 \%$ fetal calf serum (HyClone, Thermo Scientific). Human umbilical vascular endothelial cells (HUVECs) were cultured in medium 200 (Gibco, Carlsbad,

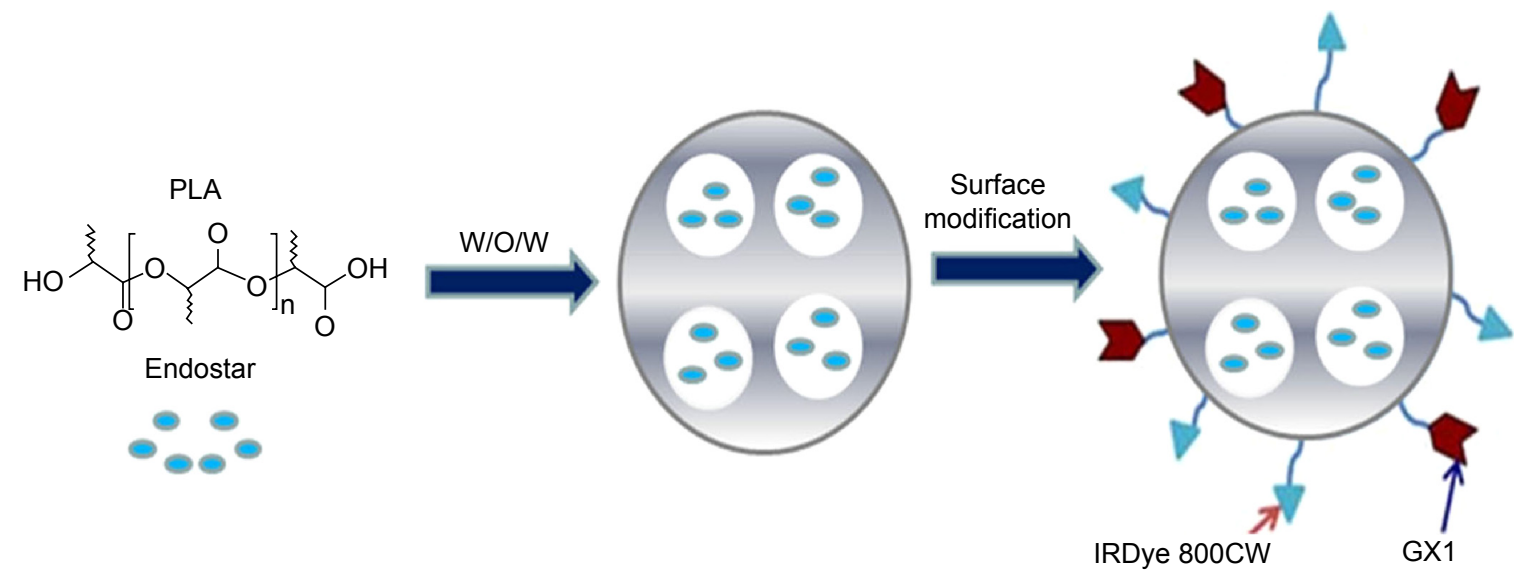

Figure I Fabrication process of GPENs. PLA-encapsulated Endostar nanoparticles were prepared through the W/O/W double-emulsion solvent evaporation method. Note: The surface of the nanoparticles was then modified with GXI peptide and IRDye $800 \mathrm{CW}$.

Abbreviations: GPENs, GXI-conjugated poly(lactic acid) nanoparticles encapsulating Endostar; PLA, polylactic acid; W/O/W, water-in-oil-in-water. 
CA, USA) and supplemented with $10 \%$ fetal calf serum. The cells were maintained at $37^{\circ} \mathrm{C}$ incubator with $5 \% \mathrm{CO}_{2}$.

\section{Cell viability assay}

HUVECs were plated in 96-well plates and treated with free Endostar and GPENs $(0.25 \mathrm{mg} / \mathrm{mL})$ for 48 hours. The cell viability was assayed by the Countess ${ }^{\circledR}$ Automated Cell Counter (Life Technologies, Grand Island, NY, USA). Trypan blue $(0.4 \%)$ was used to stain the cells.

\section{Establishment of the subcutaneous colorectal tumor-bearing mouse model}

Athymic male BALB/c nude mice (4-5 weeks old, $n=20$ ) were purchased from the Department of Experimental Animals, Peking University Health Science Center. All animal experiments were performed in accordance with the guidelines of the Institutional Animal Care and Use Committee at Peking University (Permit Number: 2011-0039). The research procedures were approved by IACUC of Peking University. The subcutaneous colorectal tumor model was established by injecting a $100 \mu \mathrm{L}$ suspension of COLO205-fLuc cells $\left(1 \times 10^{6}\right.$ cells $\left./ \mathrm{mL}\right)$ into the right upper flanks of the BALB $/ \mathrm{c}$ nude mice. The cells were allowed to grow for 2 weeks until the tumors were approximately $150 \mathrm{~mm}^{3}$ in volume.

\section{In vivo biodistribution of GPENs}

FMI and BLI were performed using the IVIS Imaging Spectrum System, and the data were analyzed by IVIS Living Imaging 3.0 software (PerkinElmer Inc., Waltham, MA, USA). FMI was utilized to examine the tumor-targeting effects and biodistribution of GPENs in vivo. The FMI data were collected 0 hour, 4 hours, 12 hours, and 24 hours after tail vein injection of GPENs (IRDye $800 \mathrm{CW}$ ) in tumor-bearing mice $(n=3)$. Free IRDye $800 \mathrm{CW}$ was used as a control $(n=3)$. The NIR dye signal and BLI signal of the tumors were obtained to evaluate GPEN biodistribution and tumor targeting. The acquisition parameters were set as $\lambda_{\mathrm{ex}}=740 \mathrm{~nm}, \lambda_{\mathrm{em}}=820 \mathrm{~nm}$, binning $=1$, and exposure time $=0.1$ seconds for FMI and $\lambda_{\mathrm{em}}=560 \mathrm{~nm}$, binning $=4$, and exposure time $=1$ second for BLI. The region of interest of the tumor was drawn to determine the fluorescence intensity, and the region of interest of the muscles in the same mouse was used as the background. Fluorescence images were normalized and reported as photons per centimeter squared per second $\left(\mathrm{p} / \mathrm{cm}^{2} / \mathrm{s}\right)$. The FMI light signal-to-noise ratio (SNR) was determined as follows:

$$
\mathrm{SNR}=\frac{\text { Fluorescence light intensity }_{\text {tumor }}}{\text { Fluorescence light intensity }_{\text {muscle }}}
$$

\section{Drug administration}

Mice with similar tumor volumes were randomly divided into three groups ( $\mathrm{n}=5$ /group) 12 days after tumor cell inoculation. The Endostar and GPEN groups were administered $10 \mathrm{mg} /$ $\mathrm{kg} /$ day of the corresponding drug via tail vein injection for 12 consecutive days. The control group was given an equal volume of $0.9 \%$ saline.

\section{Mouse body weight and tumor volume measurement}

The body weight and tumor volume of each mouse was recorded every 2 days. The tumor volume was measured by using a digital caliper and calculated according to the formula $\pi \times L \times W^{2} / 6$, where $L$ and $W$ were the respective length and width of the tumor. The tumor volume inhibition was calculated according to the following equation:

$$
\begin{gathered}
\text { Tumor volume } \\
\text { inhibition }
\end{gathered}=\left(\begin{array}{r}
\text { Volume }_{\text {Day 0 experiment group }}- \\
\text { Volume }_{\text {Day N experiment group }} \\
\text { Volume }_{\text {Day 0 control group }}- \\
\text { Volume }_{\text {Day N control group }}
\end{array}\right) \times 100 \%
$$

\section{In vivo $\mathrm{BLI}$ of subcutaneous colorectal tumor model}

BLI was implemented in the subcutaneous tumor mouse model using a small-animal optical molecular imaging system (IVIS Imaging Spectrum System, Caliper) on days 0, 3, 6,9 , and 12 of drug treatment. The mice were fasted the night before the experiment to prevent food from interfering with the imaging results. D-luciferin was dissolved and injected in PBS. The mice were anesthetized with $2 \%$ isoflurane and received intraperitoneal D-luciferin solution $(80 \mu \mathrm{L}$; $40 \mathrm{mg} / \mathrm{mL}$ ) injections 10 minutes prior to BLI. The mice were kept in a lateral position. The parameters for the BLI imaging system were binning $=4$, exposure time $=1$ second.

\section{Immunofluorescent staining of CD3 I in tumor tissues}

The tumor nodules were dissected and frozen in optimum cutting temperature compound (Leica, Wetzlar, Germany) immediately after treatment. The tumors were cryosectioned (10 $\mu \mathrm{m}$; Leica CM1950), and all slides were stored at $-80^{\circ} \mathrm{C}$ before staining. The frozen optimum cutting temperature sections were fixed in acetone for 10 minutes and stained with rat antimouse CD31 primary antibodies (BD Biosciences, Franklin Lakes, NJ, USA). Donkey antirat Alexa Fluor ${ }^{\circledR} 488$ (Invitrogen, Waltham, MA, USA) was used as a secondary 
antibody. A negative control was performed by omitting the primary antibody and incubating with secondary antibody only. The sections were washed twice and mounted with medium containing 4',6-diamidino-2-phenylindole (DAPI) (Vector Laboratories, Burlingame, CA, USA).

\section{Statistical analysis}

All experiments were repeated three times. The experimental data were presented as the mean \pm standard error of the mean from three independent experiments. One-way analysis of variance or Student's $t$-test was used to determine the statistical differences. A $P$-value $<0.05$ or $<0.01$ was considered statistically significant. Statistical analyses were performed using software Prism 4.0 (San Diego, CA, USA).

\section{Results}

\section{Characterization of GPENs}

The characteristics of the blank PNPs and GPENs were analyzed by SEM (Figure 2) and DLS. The Endostarloaded nanoparticles were spherical in structure, with a relatively smooth surface. DLS revealed that the PNPs had a mean diameter of $91.3 \pm 12.7 \mathrm{~nm}$. The PNPs' zeta potential was $-27 \pm 2.4 \mathrm{mV}$. After loading with Endostar, the diameter of the GPENs increased, with a mean diameter of $103.2 \pm 17.8 \mathrm{~nm}$, and zeta potential was $-21 \pm 3.1 \mathrm{mV}$. The size distributions coincided with the SEM images. Thus GPENs met the requirements for the enhanced permeability and retention effect, which is suitable for drug deliveryspecific tumor sites. ${ }^{31,32}$

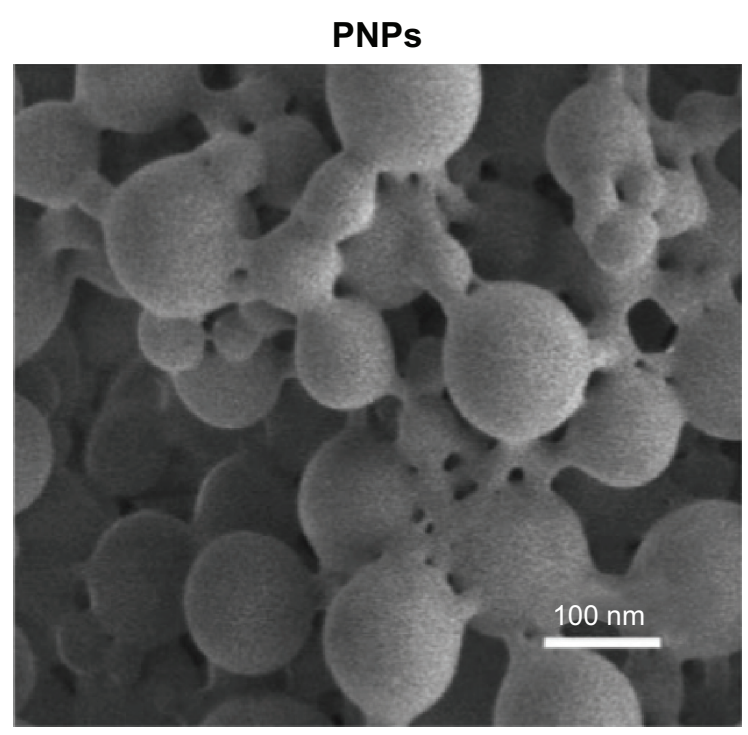

\section{In vitro Endostar release profile}

The release of Endostar from the GPENs in PBS ( $\mathrm{pH} 7.4$ and $\mathrm{pH}$ 5.0) is shown in Figure 3A. The Endostar release profile was biphasic, with an initial abrupt release and a subsequent sustained release. Under physiological conditions ( $\mathrm{pH} 7.4$ ), $20.22 \% \pm 2.23 \%$ of the loaded Endostar was released during the initial 48 hours. Almost $80 \%$ of the loaded Endostar remained enveloped in the nanoparticles after 96 hours, and the drug release was $22.67 \% \pm 1.67 \%$. In contrast, at $\mathrm{pH} 5.0$, which is similar to the tumor microenvironment, ${ }^{33}$ the release efficiency of Endostar from GPENs was slightly faster than at $\mathrm{pH}$ 7.4, reaching approximately $30.62 \% \pm 4.02 \%$ after 96 hours. Drug release capability was increased at $\mathrm{pH} 5.0$ compared to $\mathrm{pH} 7.4$, indicating that drug release was accelerated in the acidic tumor environment.

To further examine the stability of GPENs, the change in nanoparticle diameter in the presence of serum proteins was tested over 24 hours. As shown in Figure 3B, there was no obvious change in the mean diameter, indicating that the nanoparticles were stable in mouse serum.

\section{The effects of GPENs on cell viability in vitro}

We next investigated the effects of free Endostar and GPENs on HUVEC viability (Figure 4). Endostar treatment slightly reduced the cell number, and the cell viability of HUVECs was $74.75 \% \pm 4.27 \%(P<0.05)$. In contrast, GPEN treatment more effectively reduced HUVEC viability

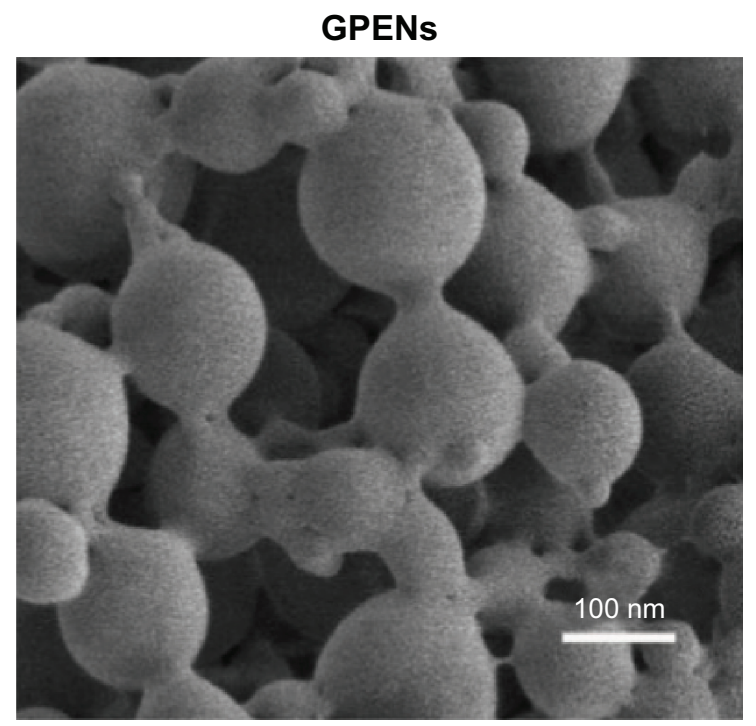

Figure 2 SEM images of the empty PNPs and GPENs. (Scale bar $=100 \mathrm{~nm}$ ).

Abbreviations: GPENs, GXI-conjugated poly(lactic acid) nanoparticles encapsulating Endostar; PLA, polylactic acid; PNPs, PLA-nanoparticles; SEM, scanning electron microscope. 


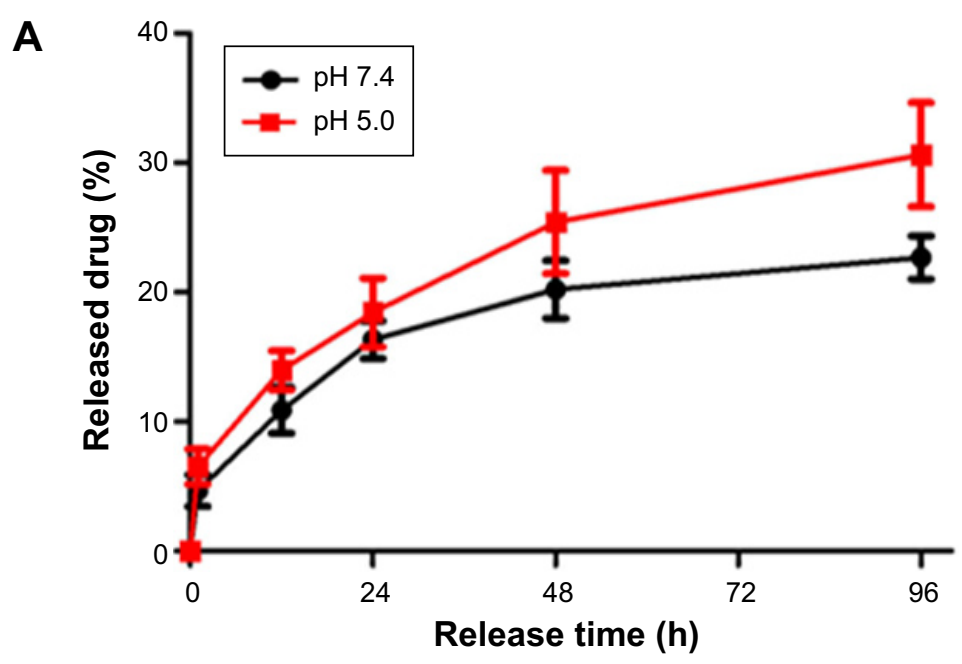

B

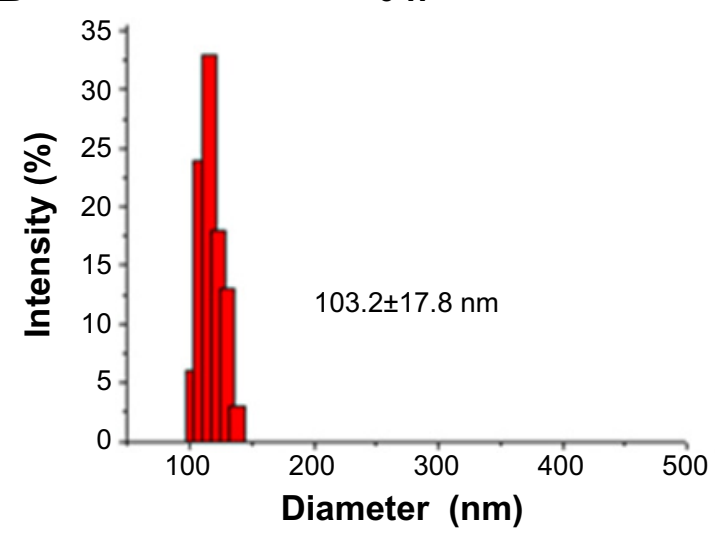

$8 \mathrm{~h}$

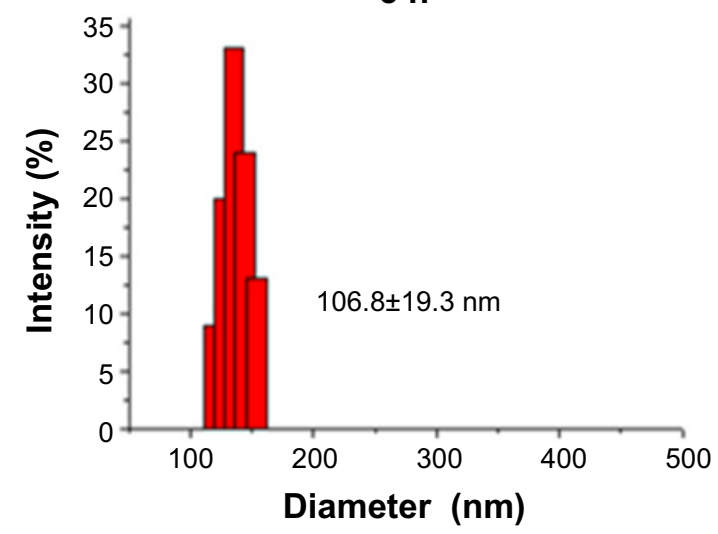

$4 \mathrm{~h}$

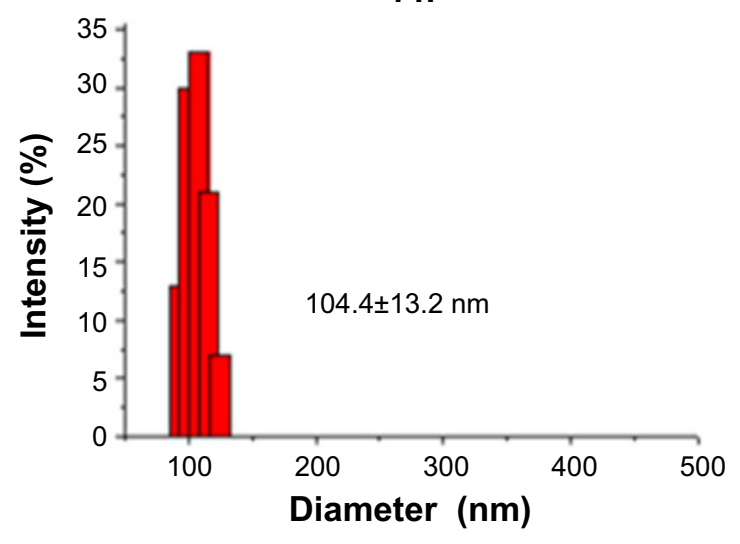

$24 \mathrm{~h}$

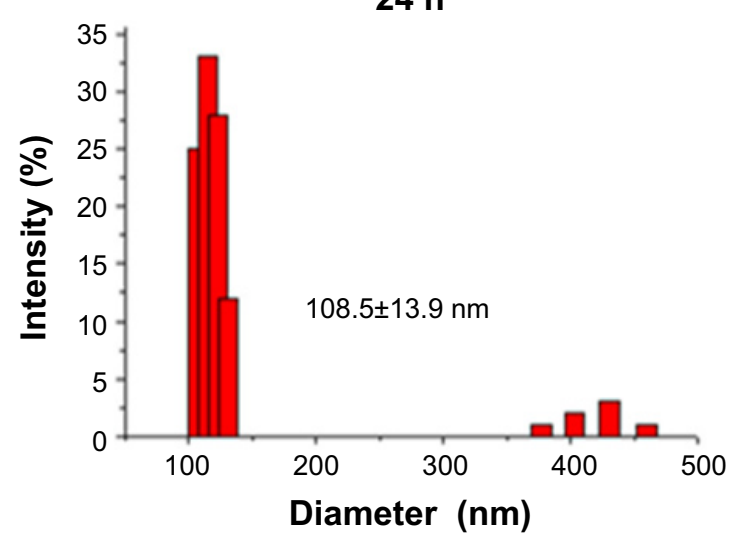

Figure 3 (A) The drug release profile from GPENs in vitro. The Endostar release profiles at $\mathrm{pH} 7.4$ and $\mathrm{pH} 5.0$ are shown in black and red, respectively. The error bars represent the mean \pm SEM. (B) The diameter changes of GPENs in $50 \%$ mouse serum over $24 \mathrm{~h}$ incubation.

Abbreviations: GPENs, GXI-conjugated poly(lactic acid) nanoparticles encapsulating Endostar; SEM, scanning electron microscope; h, hours.

$(41.25 \% \pm 0.50 \% ; P<0.01)$. Our results showed that the viability of endothelial cells treated with GPENs was significantly inhibited, as compared to the control and free Endostar, which was consistent with work by Ling et al, Xu et al, and Chen et al. ${ }^{34-36}$ These data indicated that GPENs showed a better antitumor cell effect than free Endostar in vitro and therefore possessed potential applications in antitumor treatment in vivo.

\section{Pharmacokinetics study of GPENs in vivo}

To evaluate whether GPENs enhanced tumor-targeting efficacy in vivo, GPENs (IRDye $800 \mathrm{CW}$ ) and free IRDye $800 \mathrm{CW}$ 


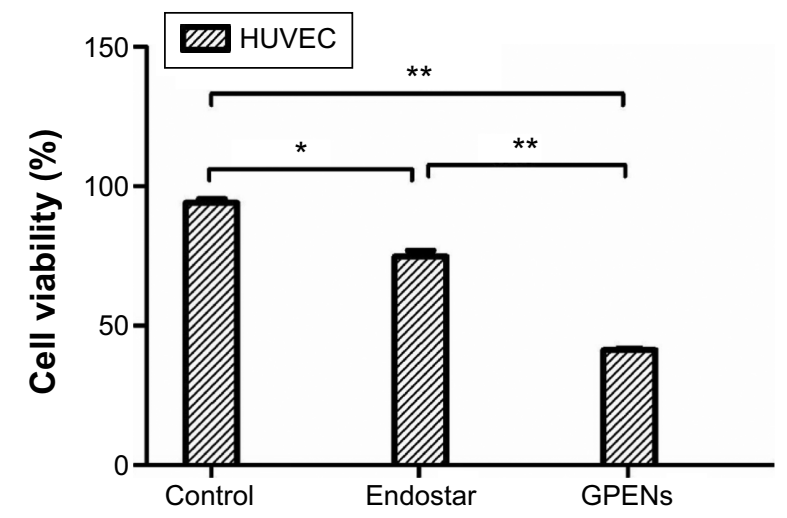

Figure 4 Cell viability assay in HUVECs after $48 \mathrm{~h}$ treatment.

Note: Data are presented as the mean \pm standard error of the mean $(* P<0.05$, $* * P<0.01)$.

Abbreviation: HUVEC, human umbilical vascular endothelial cell; h, hours; GPENs, GXI-conjugated poly(lactic acid) nanoparticles encapsulating Endostar.

were administered to colorectal tumor-bearing mice, and their in vivo biodistribution and pharmacokinetics were estimated by FMI. The BLI of COLO205-fLuc tumor-bearing nude mice following intraperitoneal injection of D-luciferin was determined to locate the tumor, and then NIR fluorescence images of COLO205-fLuc xenograft nude mice were acquired following intravenous injection of GPENs (IRDye 800CW) (Figure 5A). The GPEN probe exhibited rapid tumor targeting 12 hours after intravenous injection and maintained excellent tumor-to-background fluorescent signal contrast up to 48 hours. In contrast, there was no specific increase in signal using the free IRDye $800 \mathrm{CW}$ at the tumor site during over the 48-hour observation period. Furthermore, the signal-tonoise ratio of the GPENs at the tumor site was significantly higher from 12 hours to 48 hours compared to the free IRDye $800 \mathrm{CW}(P<0.001$; Figure 5B). These results indicated that GPENs (IRDye 800CW) possess enhanced tumor-targeting and long-acting accumulation properties, which were favorable for improving antitumor efficacy in vivo.

\section{Body weight and tumor volume changes after different treatment}

To monitor the safety of the drug treatment, the body weight was measured. During the 12-day drug treatment, no significant changes in body weight were observed among
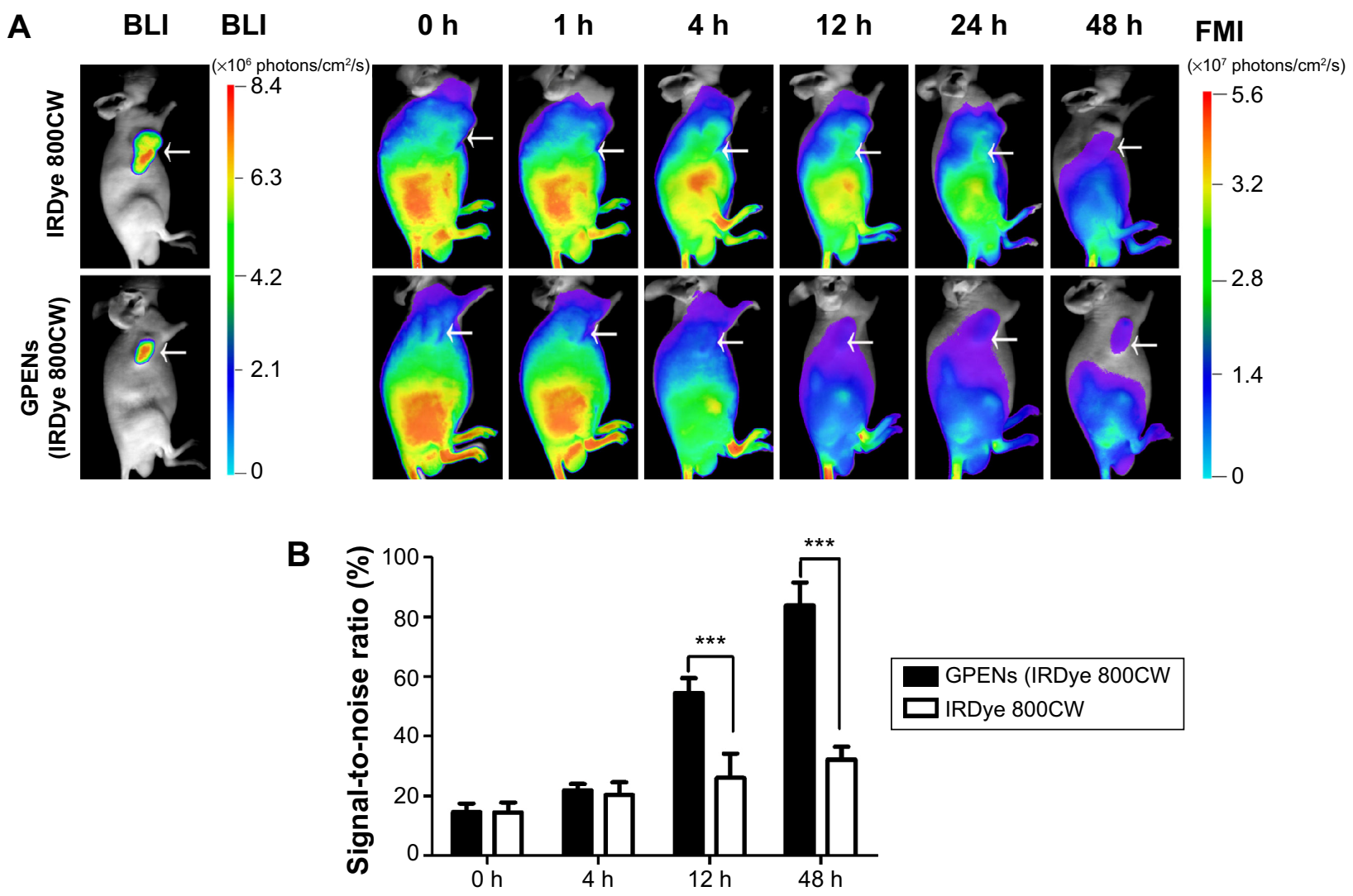

Figure 5 (A) The biodistribution of GPENs in vivo monitored by BLI and FMI. White arrows indicate the tumor region. (B) The signal-to-noise ratio of fluorescence of GPENs (IRDye $800 \mathrm{CW}$ ) and free IRDye $800 \mathrm{CW}$ at the tumor sites over a $48 \mathrm{~h}$ observation period.

Note: Data are presented as the mean \pm standard error of the mean $(* * * P<0.00 \mathrm{I})$.

Abbreviations: BLI, bioluminescence imaging; FMI, fluorescence molecular imaging; GPENs, GXI-conjugated poly(lactic acid) nanoparticles encapsulating Endostar; h, hours. 
the four groups, indicating that the dosing regimens were well tolerated and that Endostar had no obvious side effects (Figure 6A).

To compare the drug treatment effects in different groups, the tumor volume was measured using a digital caliper, and inhibition was calculated and compared. The results indicated that tumor volume growth was significantly inhibited by GPEN treatment, as compared to the control and the free Endostar groups (Figure 6B). The data also suggested that targeted treatment using GPENs increased the antitumor effect of Endostar.

\section{Monitoring tumor growth with different drug treatments through $\mathrm{BLI}$ in vivo}

In order to fully examine the antitumor effects of GPENs in a sensitive way, BLI optical molecular imaging was performed on the orthotopic colorectal tumor-bearing mice during the 12-day treatment. The nude mice bearing colorectal tumors displayed strong BLI signals in the tumor regions. In general, the BLI intensity greatly increased in the control group over the 12-day observation (Figure 7A-D), whereas the light intensity increased relatively slowly in the free Endostar treatment group (Figure 7E-H). The inhibitory effect was even more dramatic with GPEN treatment compared to free Endostar treatment (Figure 7I-L). After the 12-day treatment period, the average BLI intensity of the control group and Endostar group was $150.54 \pm 39.96 \times 10^{6}$ photons $/ \mathrm{s} / \mathrm{cm}^{2}$ and $109.22 \pm 36.83 \times 10^{6}$ photons $/ \mathrm{s} / \mathrm{cm}^{2}$, respectively $(P<0.05)$. In contrast, the average BLI intensity value was only $42.37 \pm 21.03 \times 10^{6}$ photons $/ \mathrm{s} / \mathrm{cm}^{2}$ in the GPEN treatment group $(P<0.01$; Figure 8). These data supported the prominent and safe antitumor efficacy of GPENs in vivo.

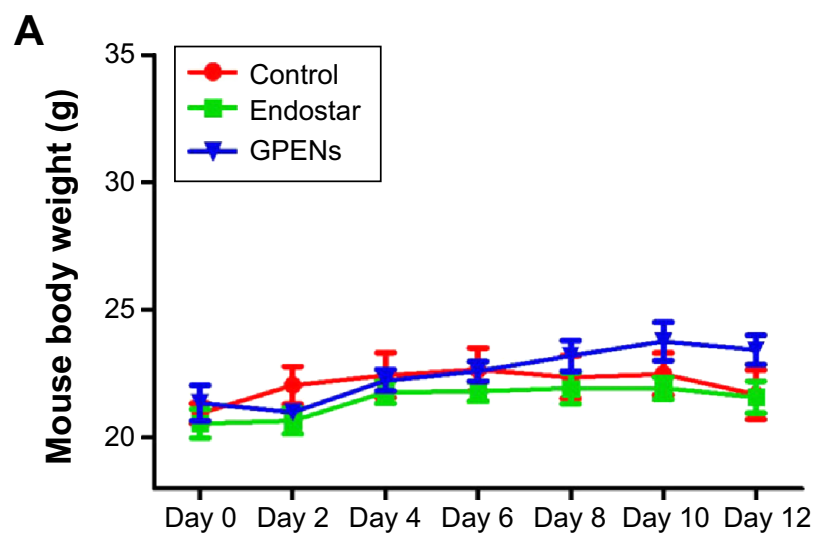

\section{Immunohistological examination of the effects of GPENs on tumor angiogenesis}

To further confirm the in vivo antitumor effects, tumor nodules were dissected at the end of the drug treatment and immunohistochemically stained for CD31, a marker of microvascular endothelial cells. The CD31 expression was intense in the control group (Figure 9A) and was decreased in the two treated groups (Figure 9B, C). Lower CD31 expression was observed in the Endostar-treated group (Figure 9B), and the CD31 expression level was the lowest in the GPEN treatment group (Figure 9C). The nuclei were stained using DAPI (Figure 9D-F), and merged images of CD31 and DAPI are also presented (Figure 9G-I). The CD31 immunofluorescent staining results support the hypothesis that GPENs displayed enhanced antitumor angiogenesis efficacy compared to other two groups in vivo.

\section{Discussion}

Antiangiogenic therapy could be improved through coupling with specific molecular markers that target the tumor vasculature. In this study, we developed a novel angiogenesistargeting theranostic nanoparticle by conjugating the angiogenesis-targeting peptide GX1 to the PLA nanosystem encapsulating Endostar and labeling with the NIR fluorescent dye IRDye $800 \mathrm{CW}$. Our data demonstrated that these nanoparticles successfully delivered Endostar to the tumor regions and displayed enhanced antitumor efficacy. In vitro cell proliferation experiments revealed enhanced inhibition of HUVEC proliferation following GPEN treatment compared to free Endostar treatment. With the aid of molecular imaging, including FMI and BLI, the in vivo biodistribution of GPENs and the antitumor treatment efficacy were dynamically and

B

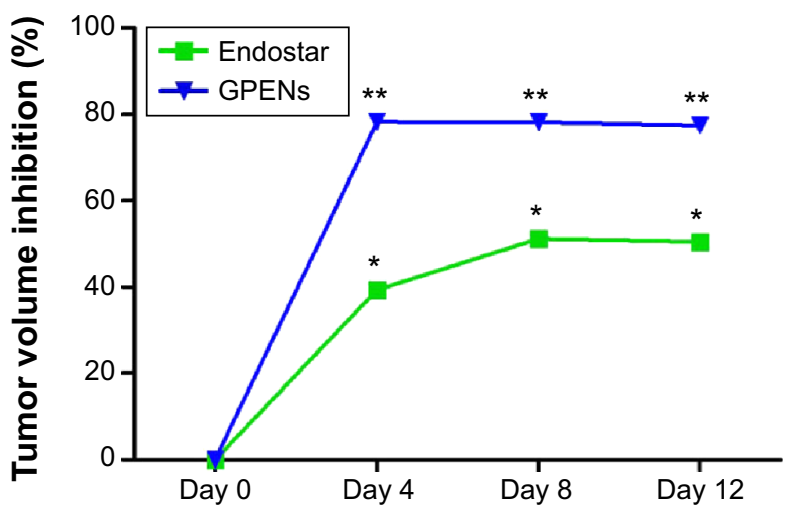

Figure 6 Mouse body weight and tumor volume changes over 12 days.

Notes: The average mouse body weight $(\mathbf{A})$ and tumor volume inhibition $(\mathbf{B})$ in mice treated with control, free Endostar, and GPENs. Data are presented as the mean \pm standard error of the mean $(* P<0.05 ; * * P<0.01)$.

Abbreviation: GPENs, GXI-conjugated poly(lactic acid) nanoparticles encapsulating Endostar. 

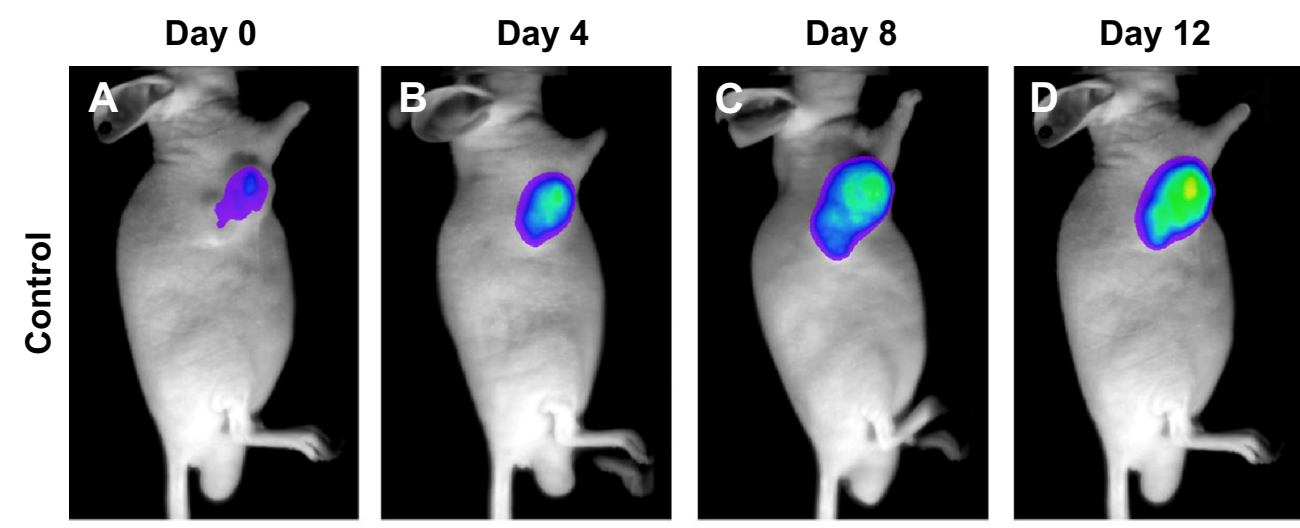

$\times 10^{6}$

$\mathrm{p} / \mathrm{s} / \mathrm{cm}^{2}$
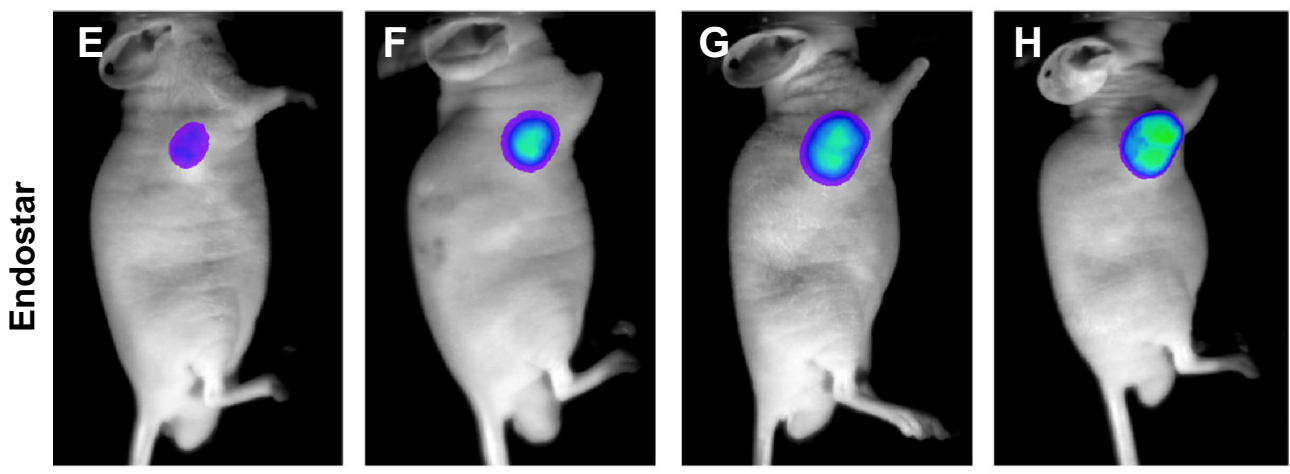

6.3
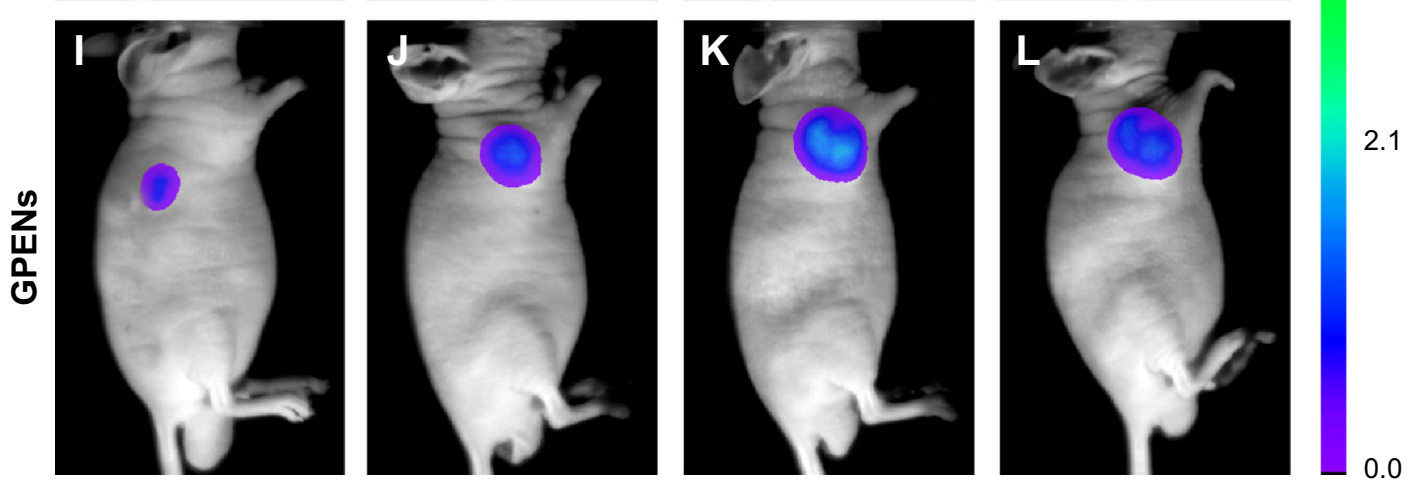

Figure 7 Representative BLI of tumors in vivo over 12 days observation.

Notes: Nude mice bearing colon tumors derived from the COLO205-fLuc cell line displayed BLI, and the signal intensities reflected the tumor growth condition and drug treatment efficacy in vivo. The BLI of colorectal tumors with different treatment included the control group (A-D), Endostar treatment (E-H), and GPENs treatment (I-L).

Abbreviations: BLI, bioluminescence imaging; GPENs, GXI-conjugated poly(lactic acid) nanoparticles encapsulating Endostar.

noninvasively monitored. Moreover, the in vivo antiangiogenesis efficacy was further validated through CD31 immunohistological examination of tumor tissues.

Tumor angiogenesis is a key requirement for cancer progression, and without angiogenesis, the tumor may not grow beyond a few millimeters in diameter. ${ }^{37,38}$ Advanced peptide-based fluorescence molecular probes targeting angiogenesis are being intensely investigated to improve antitumor angiogenesis therapy. The GX1 peptide (sequence: CGNSNPKSC) was previously identified as an angiogenesis target through in vivo screening using a phage-display peptide library. Studies revealed that the GX1 peptide specifically binds to human gastric cancer vasculature. ${ }^{39,40}$ Immunohistochemical staining, enzyme-linked immunosorbent assay, and immunofluorescence studies indicated that GX1 could be used as a novel vascular marker for human cancers. ${ }^{41}$ Therefore, in our study, the GX1 peptide was conjugated to PNPs encapsulating Endostar, and the nanoparticles were characterized in vitro and in vivo. SEM images showed that the diameter of the GPENs was around $100 \mathrm{~nm}$ (Figure 2), which met the requirement for an enhanced permeability and retention effect at the tumor site. In addition, the particle sizes could be maintained stably in serum for 24 hours (Figure 3B), which was favorable for in vivo administration 


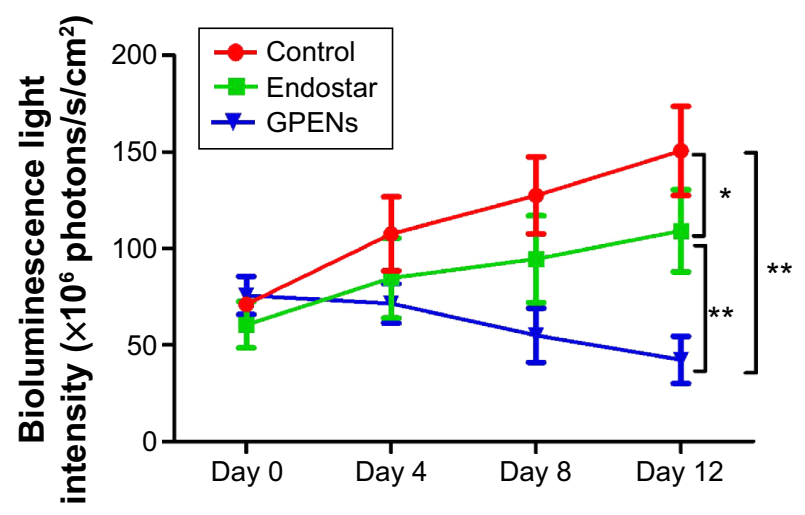

Figure $8 \mathrm{BLI}$ changes in the tumors of the control, Endostar, and GPEN groups during the 12-day observation period.

Note: Data are presented as the mean \pm standard error of mean $\left({ }^{*} P<0.05\right.$, $\left.{ }^{* *} P<0.01\right)$. Abbreviations: BLI, bioluminescence imaging; GPENs, GXI-conjugated poly(lactic acid) nanoparticles encapsulating Endostar. and treatment. Furthermore, the drug release data showed that there was more Endostar released at $\mathrm{pH}$ 5.0, which is similar to the $\mathrm{pH}$ of the tumor microenvironment, ${ }^{33}$ compared to $\mathrm{pH}$ 7.4 , suggesting intelligently controlled release of drugs and potential enhancement of antitumor efficacy with the GPENs (Figure 3A).

Noninvasive optical molecular imaging strategies can be utilized to observe the onset and progression of neoplastic transformations at the molecular and cellular levels, thus allowing us to detect the tumor in a more sensitive and dynamic way. ${ }^{42}$ Therefore, we tested whether conjugating the GX1 with IRDye $800 \mathrm{CW}$ could facilitate optical imaging of the tumor in vivo. By conjugating the IRDye $800 \mathrm{CW}$, the biodistribution and tumor-targeting effects of the
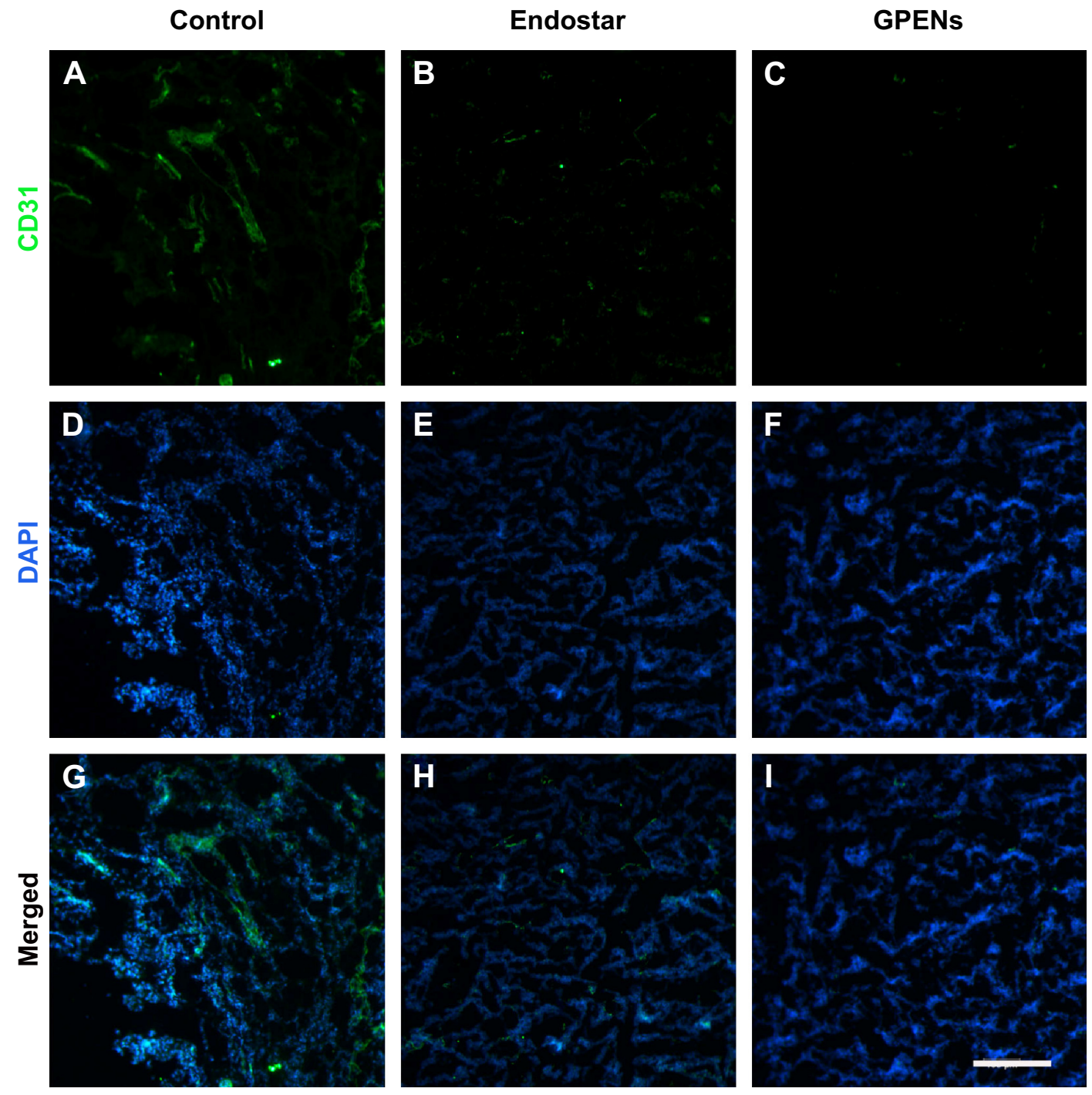

Figure 9 CD3 I immunofluorescent staining of tumor sections after 12 days of continuous treatment.

Notes: $C D 3$ I is a microvascular marker and was stained to evaluate the antiangiogenic effects of different drug treatments. The CD3। expression was shown (A-C) CD3 I expression showing green color; (D-F) the nuclear staining DAPI showing blue color; $(\mathbf{G}-\mathbf{I})$ merged images of both CD3I and DAPI (Scale bar $=200 \mu \mathrm{m}, \mathrm{I0} \times$ ). Abbreviation: GPENs, GXI-conjugated poly(lactic acid) nanoparticles encapsulating Endostar. 
nanoparticles could be monitored throughout the therapeutic process in vivo. The in vivo FMI data showed that GPENs (IRDye $800 \mathrm{CW}$ ) rapidly targeted the tumor 12 hours after injection and maintained excellent tumor-to-background fluorescent signal contrast for 48 hours. In contrast, there was no significant accumulation of free IRDye $800 \mathrm{CW}$ at the tumor sites (Figure 5). These results suggested that the GX1 peptide facilitated tumor-specific targeting efficacy, which was favorable for improving the antitumor treatment efficacy and reducing systemic side effects of chemotherapy in vivo.

Antiangiogenic therapy is an emerging research front and a new clinical strategy in tumor-targeting therapy. ${ }^{43,44}$ Endostar, a novel recombinant human endostatin, has been shown to suppress tumor growth effectively by inhibiting angiogenesis. ${ }^{45,46}$ Endostar has a broad spectrum of activity against solid tumors through the inhibition of endothelial cell proliferation, migration, and vessel formation. Nevertheless, to bring antiangiogenesis therapeutics into clinical practice, selective infiltration and controlled release of drugs at tumor sites must be improved. Hence, we further examined whether GPENs improved antitumor activity. The in vivo therapeutic efficacy of GPENs was evaluated in a subcutaneous colorectal tumor-bearing mouse model. The treatment effects were dynamically monitored not only by measuring the tumor volumes by digital caliper (Figure 6) but also by utilizing BLI (Figure 7). In this study, the BLI light intensity analysis showed that compared to Endostar, GPENs improved tumor growth inhibition; this finding was consistent with the data of tumor volume measurement (Figure 8). Furthermore, we performed in vitro immunohistochemistry staining for CD31, a marker of microvascular endothelial cells. The data indicated that microvascular density was decreased in the GPEN-treated group (Figure 9). Together, the data suggested that GPENs improved the antitumor activity via the inhibition of tumor angiogenesis in vivo.

Moreover, the mice in the control and drug-treated groups showed no abnormal behavior, and the body weight increased steadily during the 12-day period. These data suggested that Endostar and GPENs did not cause obvious side effects.

\section{Conclusion}

GX1, a tumor vasculature endothelium-specific ligand, played a role in cancer-associated angiogenesis. We successfully developed GPENs, a novel theranostic drug platform and showed that they improve in vivo colorectal tumor treatment efficacy compared to free Endostar. Additionally, conjugation of GPENs with IRDye $800 \mathrm{CW}$ dye was suitable for targeted imaging and therapy of CRC in vivo. Such targeted, fluorescently labeled drugs combined with probes may serve as novel theranostic agents for highly selective detection and treatment of CRC. Our findings may be useful for the development of a novel, preclinical treatment regimen and may inform the design and management of future clinical trials.

\section{Acknowledgments}

This work was supported by the National Basic Research Program of China (973) (under grants 2011CB707702, 2014CB748600, and 2015CB755500) and the National Natural Science Foundation of China (81227901 and 81470083). This work was supported in part by the State Key Laboratory of Management and Control for Complex Systems (under grant no Y3S9021F30).

\section{Disclosure}

The authors report no conflicts of interest in this work.

\section{References}

1. Motzer RJ, Nosov D, Eisen T, et al. Tivozanib versus sorafenib as initial targeted therapy for patients with advanced renal cell carcinoma: results from a phase III randomized, open-label, multicenter trial. J Clin Oncol. 2012;30(15):277S

2. Brannon-Peppas L, Blanchette JO. Nanoparticle and targeted systems for cancer therapy. Adv Drug Deliv Rev. 2012;64:206-212.

3. Zhang Z, Lee JC, Lin L, et al. Activation of the AXL kinase causes resistance to EGFR-targeted therapy in lung cancer. Nat Genet. 2012;44(8) $852-860$.

4. Weis SM, Cheresh DA. Tumor angiogenesis: molecular pathways and therapeutic targets. Nat Med. 2011;17(11):1359-1370.

5. Weidner N, Semple JP, Welch WR, Folkman J. Tumor angiogenesis and metastasis - correlation in invasive breast carcinoma. $N$ Engl $J$ Med. 1991;324(1):1-8.

6. Breitbach CJ, Arulanandam R, De Silva N, et al. Oncolytic vaccinia virus disrupts tumor-associated vasculature in humans. Cancer Res. 2013;73(4):1265-1275.

7. Choueiri TK, Xie W, Kollmannsberger C, et al. The impact of cytoreductive nephrectomy on survival of patients with metastatic renal cell carcinoma receiving vascular endothelial growth factor targeted therapy. J Urol. 2011;185(1):60-66.

8. Curnis F, Sacchi A, Longhi R, Colombo B, Gasparri A, Corti A. IsoDGR-tagged albumin: A new $\alpha v \beta 3$ selective carrier for nanodrug delivery to tumors. Small. 2012;9(5):673-678.

9. Hong H, Yang K, Zhang Y, et al. In vivo targeting and imaging of tumor vasculature with radiolabeled, antibody-conjugated nanographene. ACS Nano. 2012;6(3):2361-2370.

10. Schmieder AH, Winter PM, Williams TA, et al. Molecular MR imaging of neovascular progression in the $\mathrm{Vx} 2$ tumor with $\alpha \mathrm{v} \beta 3$-targeted paramagnetic nanoparticles. Radiology. 2013;268(2):470-480.

11. Sugahara KN, Teesalu T, Karmali PP, et al. Coadministration of a tumor-penetrating peptide enhances the efficacy of cancer drugs. Science. 2010;328(5981):1031-1035.

12. Chen K, Sun X, Niu G, et al. Evaluation of $64 \mathrm{Cu}$ labeled GX1: a phage display peptide probe for PET imaging of tumor vasculature. Mol Imaging Biol. 2012;14(1):96-105. 
13. Kenter GG, Welters MJ, Valentijn AR, et al. Phase I immunotherapeutic trial with long peptides spanning the E6 and E7 sequences of high-risk human papillomavirus 16 in end-stage cervical cancer patients shows low toxicity and robust immunogenicity. Clin Cancer Res. 2008;14(1):169-177.

14. Chen B, Cao S, Zhang Y, et al. A novel peptide (GX1) homing to gastric cancer vasculature inhibits angiogenesis and cooperates with TNF alpha in anti-tumor therapy. BMC Cell Biol. 2009;10(1):63-73.

15. Wu K, Nie Y, Guo C, Chen Y, Ding J, Fan D. Molecular basis of therapeutic approaches to gastric cancer. J Gastroenterol Hepatol. 2009;24(1): $37-41$.

16. Chen K, Yap LP, Park R, et al. A Cy5. 5-labeled phage-displayed peptide probe for near-infrared fluorescence imaging of tumor vasculature in living mice. Amino Acids. 2012;42(4):1329-1337.

17. Ling Y, Yang Y, Lu N, et al. Endostar, a novel recombinant human endostatin, exerts antiangiogenic effect via blocking VEGF-induced tyrosine phosphorylation of KDR/Flk-1 of endothelial cells. Biochem Biophys Res Commun. 2007;361(1):79-84.

18. Xu HX, Huang XE, Qian ZY, Xu X, Li Y, Li CG. Clinical observation of Endostar ${ }^{\circledR}$ combined with chemotherapy in advanced colorectal cancer patients. Asian Pac J Cancer Prev. 2011;12(11): 3087-3090

19. Chen W, Hu S. Suitable carriers for encapsulation and distribution of Endostar: comparison of Endostar-loaded particulate carriers. Int $J$ Nanomedicine. 2011;2011(6):1535-1541.

20. Chen X, Gambhir SS, Cheon J. Theranostic nanomedicine. Acc Chem Res. 2011;44(10):1029-1038.

21. McCarthy JR. The future of theranostic nanoagents. Nanomedicine. 2009;4(7):693-695.

22. James ML, Gambhir SS. A molecular imaging primer: modalities, imaging agents, and applications. Physiol Rev. 2012;92(2):897-965.

23. Weissleder R. Molecular imaging in cancer. Science. 2006;312(5777): 1168-1171.

24. Weissleder R, Pittet MJ. Imaging in the era of molecular oncology. Nature. 2008;452(7187):580-589.

25. Liu Z, Liu S, Niu G, Wang F, Liu S, Chen X. Optical imaging of integrin $\alpha \mathrm{v} \beta 3$ expression with near-infrared fluorescent RGD dimer with tetra (ethylene glycol) linkers. Mol Imaging. 2010;9(1):21-29.

26. Kaufmann BA, Carr CL, Belcik JT, et al. Molecular imaging of the initial inflammatory response in atherosclerosis implications for early detection of disease. Arterioscler Thromb Vasc Biol. 2010;30(1):54-59.

27. Troyan SL, Frangioni JV. The FLARETM intraoperative nearinfrared fluorescence imaging system: a first-in-human clinical trial in breast cancer sentinel lymph node mapping. Ann Surg Oncol. 2009;16(10):2943-2952.

28. Jang B, Park JY, Tung CH, Kim IH, Choi Y. Gold nanorod-photosensitizer complex for near-infrared fluorescence imaging and photodynamic/ photothermal therapy in vivo. ACS Nano. 2011;5(2):1086-1094.

29. Hilderbrand SA, Weissleder R. Near-infrared fluorescence: application to in vivo molecular imaging. Curr Opin Chem Biol. 2010;14(1): 71-79.
30. Sevick-Muraca E. Translation of near-infrared fluorescence imaging technologies: emerging clinical applications. Annu Rev Med. 2012;63: 217-231.

31. Prabhakar U, Maeda H, Jain RK, et al. Challenges and key considerations of the enhanced permeability and retention effect for nanomedicine drug delivery in oncology. Cancer Res. 2013;73(8):2412-2417.

32. Bala V, Rao S, Boyd BJ, Prestidge CA. Prodrug and nanomedicine approaches for the delivery of the camptothecin analogue SN38. J Control Release. 2013;172(1):48-61.

33. Park JH, Cho HJ, Yoon HY, et al. Hyaluronic acid derivative-coated nanohybrid liposomes for cancer imaging and drug delivery. J Control Release. 2014;174:98-108.

34. Ling Y, Lu N, Gao Y, et al. Endostar induces apoptotic effects in HUVECs through activation of caspase-3 and decrease of Bcl-2. Anticancer Res. 2009;29(1):411-417.

35. Xu X, Mao W, Chen Q, et al. Endostar, a modified recombinant human endostatin, suppresses angiogenesis through inhibition of Wnt/ $\beta$-catenin signaling pathway. PLoS One. 2014;9(9):e107463.

36. Chen $\mathrm{B}, \mathrm{Cao} \mathrm{S}, \mathrm{Z}$ hang $\mathrm{Y}$, et al. A novel peptide (GX1) homing to gastric cancer vasculature inhibits angiogenesis and cooperates with TNF alpha in anti-tumor therapy. BMC Cell Biol. 2009;10:63-73.

37. Bergers G, Benjamin LE. Tumorigenesis and the angiogenic switch. Nat Rev Cancer. 2003;3(6):401-410.

38. Folkman J. Angiogenesis in cancer, vascular, rheumatoid and other disease. Nat Med. 1995;1(1):27-31.

39. Cao S, Liu Y, Li X, et al. Expression, purification, and characterization of recombinant protein GX1-rmhTNFalpha. Mol Biotechnol. 2009;43(1): $1-7$.

40. $\mathrm{Hu} \mathrm{H}$, Yin J, Wang M, et al. GX1 targeting delivery of rmhTNFalpha evaluated using multimodality imaging. Int J Pharm. 2013;461(1): 181-191.

41. Hui X, Han Y, Liang S, et al. Specific targeting of the vasculature of gastric cancer by a new tumor-homing peptide CGNSNPKSC. J Control Release. 2008;131(2):86-93.

42. Hsu AR, Hou LC, Veeravagu A, et al. In vivo near-infrared fluorescence imaging of integrin $\alpha v \beta 3$ in an orthotopic glioblastoma model. Mol Imaging Biol. 2006;8(6):315-323.

43. Hu YL, Jahangiri A, Delay M, Aghi MK. Tumor cell autophagy as an adaptive response mediating resistance to treatments such as antiangiogenic therapy. Cancer Res. 2012;72(17):4294-4299.

44. Stoll BR, Migliorini C, Kadambi A, Munn LL, Jain RK. A mathematical model of the contribution of endothelial progenitor cells to angiogenesis in tumors: implications for antiangiogenic therapy. Blood. 2003;102(7):2555-2561.

45. Li XQ, Shang BY, Wang DC, Zhang SH, Wu SY, Zhen YS. Endostar, a modified recombinant human endostatin, exhibits synergistic effects with dexamethasone on angiogenesis and hepatoma growth. Cancer Lett. 2011;301(2):212-220.

46. Fu XH, Li J, Zou Y, et al. Endostar enhances the antineoplastic effects of combretastatin A4 phosphate in an osteosarcoma xenograft. Cancer Lett. 2011;312(1):109-116.
International Journal of Nanomedicine

\section{Publish your work in this journal}

The International Journal of Nanomedicine is an international, peerreviewed journal focusing on the application of nanotechnology in diagnostics, therapeutics, and drug delivery systems throughout the biomedical field. This journal is indexed on PubMed Central, MedLine, CAS, SciSearch ${ }^{\circledR}$, Current Contents ${ }^{\circledR} /$ Clinical Medicine,
Dovepress

Journal Citation Reports/Science Edition, EMBase, Scopus and the Elsevier Bibliographic databases. The manuscript management system is completely online and includes a very quick and fair peer-review system, which is all easy to use. Visit http://www.dovepress.com/ testimonials.php to read real quotes from published authors. 